\title{
Correction to: Soluble Guanylate Cyclase Stimulators and Activators
}

Peter Sandner, Daniel P. Zimmer, G. Todd Milne, Markus Follmann, Adrian Hobbs, and Johannes-Peter Stasch

\section{Correction to: Online First Chapter "Soluble Guanylate Cyclase Stimulators and Activators" in book series Handbook of Experimental Pharmacology, https://doi.org/10.1007/164_2018_197}

The chapter 'Soluble Guanylate Cyclase Stimulators and Activators' has now been made available open access under a CC BY 4.0 license. 